\title{
Application of Computer Simulation Modeling to Medication Administration Process Redesign
}

\author{
Nathan Huynh, $\mathbf{P h D}^{1 *}$, Rita Snyder, $\mathbf{P h D}, \mathbf{R N}^{2}$, Jose M. Vidal, $\mathrm{PhD}^{3}$, \\ Abbas S. Tavakoli, DrPH, MPH, $\mathrm{ME}^{2}$ and Bo Cai, $\mathrm{PhD}^{4}$ \\ ${ }^{1}$ Civil \& Environmental Engineering, \\ ${ }^{3}$ Computer Science and Engineering, College of Engineering and Computing; \\ ${ }^{2}$ College of Nursing; \\ ${ }^{4}$ Epidemiology and Biostatistics, Arnold School of Public Health, \\ University of South Carolina, Columbia, SC USA
}

Submitted November 2011. Accepted for publication June 2012.

\begin{abstract}
The medication administration process (MAP) is one of the most high-risk processes in health care. MAP workflow redesign can precipitate both unanticipated and unintended consequences that can lead to new medication safety risks and workflow inefficiencies. Thus, it is necessary to have a tool to evaluate the impact of redesign approaches in advance of their clinical implementation. This paper discusses the development of an agent-based MAP computer simulation model that can be used to assess the impact of MAP workflow redesign on MAP performance. The agent-based approach is adopted in order to capture Registered Nurse medication administration performance. The process of designing, developing, validating, and testing such a model is explained. Work is underway to collect MAP data in a hospital setting to provide more complex MAP observations to extend development of the model to better represent the complexity of MAP.
\end{abstract}

Keywords: medication administration process, agent-based computer simulation, clinical process redesign

\section{INTRODUCTION}

Medication errors in the United States constitute a serious safety risk that result in approximately 7,000 deaths, harm to approximately 1.5 million people, and billions of dollars in hospital treatment costs annually [1-4]. The majority of medication errors are precipitated by fragmented and highly complex medication management processes [5]. Cognition literature indicates that process fragmentation from frequent task switching increases the performer's mental burden [6]. In hospital settings, frequent task switching often increases physical activities that can precipitate interruptions and result in a higher

\footnotetext{
*Corresponding Author: Nathan Huynh, PhD, Civil \& Environmental Engineering, College of Engineering and Computing, University of South Carolina, 300 Main Street, Columbia, SC 29208. Phone: (803) 7778947. E-mail: huynhn@cec.sc.edu. Other authors: ritsny520@aol.com; jmvidal@gmail.com; atavakoli@ mailbox.sc.edu; bocai@mailbox.sc.edu.
} 
likelihood of cognitive slips and mistakes [6]. This is especially true for the medication administration process (MAP) that includes numerous disjointed tasks that must be accomplished while maintaining the overall quality and safety of patient care [7]. The increasingly complex MAP is a key process redesign target [8]. Factors that contribute to MAP complexity include escalating patient acuity levels, numerous generic and trade medication names, expanded medication delivery routes, increased use of new and diverse medication safety technology, and increased medication orders [9].

In recent years, MAP redesign efforts have increased to reduce risk and improve efficiency [10-11]. The Institute of Medicine has encouraged development of reliable and valid redesign approaches that contribute to a basic understanding of current health care processes and provide insight into the potential impact of proposed redesign solutions [12-14]. The use of computer simulation provides a unique opportunity to model the impact of redesign approaches in advance of their clinical implementation. This has several advantages: (1) it supports a low risk, systematic and controlled redesign approach; (2) it increases the likelihood of pre-implementation identification of unintended and unanticipated redesign consequences thus reducing redesign risk; (3) it actively engages clinicians as partners in the modeling and redesign process thus broadening the potential for inclusion of critical clinical workflow and safety factors; and (4) it supports ongoing formative evaluation during the redesign process to assess the impact of redesign factors on the process as a whole.

A growing number of studies have utilized computer simulation modeling to analyze and improve clinical processes. More recent applications of computer simulation in health care process redesign have used the agent-based modeling approach. An advantage of agent-based modeling is the ability to represent human behavior in simulation models. Sibbel and Urban demonstrated that agent-based modeling is more appropriate for economic and organizational planning for hospital management than the traditional modeling approaches where staffs are treated as passive resources [15]. Kanagarajah et al. discussed an agent-based modeling framework to study health care system improvements and their impact on patient safety, economics and workloads [16]. They concluded that agent-based modeling offers a feasible framework to analyze complex adaptive behavior of a health care system.

This paper focuses on modeling Registered Nurse (RN) MAP workflow. It examines MAP fragmentation and interruptions to identify potential ways to improve medication administration safety. The agent-based modeling approach is applied using MAP workflow data collected from a prior pilot study. The objectives of this paper are to (1) describe the application of agent-based modeling techniques to the development of a computer simulation model of the MAP, and (2) demonstrate the potential use of the model in MAP redesign.

\section{METHODS}

The agent-based computer simulation MAP model was developed in two phases. Phase one involved generating baseline MAP observation data for computer simulation model development [19]. Phase two involved MAP computer simulation model design, development, validation, and testing. 


\subsection{Baseline Data Generation for Computer Simulation Modeling}

The following sections provide a brief summary of the previous pilot study [17] and data analyses performed to generate baseline data for the MAP computer simulation model.

\subsubsection{Pilot Study Summary}

A pilot study was conducted in a clinical simulation lab (CSL) over a period of 4 weeks. Three RNs were recruited from a local medical center to model the oral MAP; the study team obtained IRB approval for the study, and RNs signed consent forms. Each RN was asked to perform the MAP in the same way that they would normally carry it out in their regular hospital clinical practice. Each RN modeled the MAP 3 times for a total of 9 combined sessions per RN for a grand total of 27 MAP sessions. All MAP sessions were video recorded.

\subsubsection{MAP Data Analyses}

The objective of the data analysis was to uncover hidden regularities embedded in MAP workflow patterns. The analysis used two pattern recognition techniques: consecutive sequential pattern analysis (CSPA) and transition probability analysis (TPA). The CSPA searched for workflow segments that reoccurred frequently both within and across observations. The TPA computed probabilities for transitioning among pairs of MAP tasks. The workflow quantifier "average continuous time" (ACT) proposed by Zheng et al. [6] was also used to assess the magnitude of workflow fragmentation. ACT is the average amount of time continuously spent on performing clinical activities belonging to the same MAP function while workflow fragmentation is the rate at which clinicians switch between tasks. The pilot study produced 27 workflow patterns with a total of 70 discrete MAP tasks and interruptions as shown in Table 1. When an RN is at the computer performing an activity, this event may be recorded as two separate tasks such as "At computer" and "Log-in to MAR." Each discrete task is provided in Table 1. The function associated with each task is also provided.

Baseline MAP workflow fragmentation was assessed using a timeline belt approach. This approach was proposed by Zheng et al. [6] as a method to more easily visualize workflow fragmentation based on various process functions and tasks. As depicted in Figure 1, each timeline belt or row represents one of the 27 MAP sessions. For each timeline belt, MAP tasks and interruptions were mapped onto one of four MAP functions: preparation (yellow), administration (green), documentation (red), and interruption (blue). The length of a colored stripe is proportional to how long the task or interruption lasted in seconds. The number of tasks performed per session varied between 16 and 30. As demonstrated in Figure 1, RNs frequently switched between MAP functions and tasks, and experienced some interruptions. The frequency at which RNs switched from one task to another provided an important behavioral characteristic that is central to the agent-based modeling approach.

Figure 2 shows the average amount of time an RN spent continuously performing tasks associated with a specific MAP function and an error bar for that function indicating the value of one standard deviation. Of the four primary MAP tasks, 
Table 1. MAP tasks and interruptions list

\begin{tabular}{|c|c|c|c|c|c|}
\hline ID & Task Description & Function & ID & Description & Function \\
\hline 1 & Greet patient & Prepare & 36 & Walk out of room & Document \\
\hline 2 & Pre-wash hands & Prepare & 37 & Explain procedure & Prepare \\
\hline 3 & Chat with patient & Prepare & 38 & Position patient & Admin \\
\hline 4 & Scan patient ID band & Prepare & 39 & Assess any other needs & Admin \\
\hline 5 & $\begin{array}{l}\text { Log-in to medication } \\
\text { administration record (MAR) }\end{array}$ & Prepare & 40 & Document MAR & Document \\
\hline 6 & Review MAR & Prepare & 41 & Pour water & Admin \\
\hline 7 & Obtain medications & Prepare & 42 & Assess patient's condition & Admin \\
\hline 8 & Verify correct medications & Prepare & 43 & Point of care & Admin \\
\hline 9 & $\begin{array}{l}\text { Check medication expiration } \\
\text { date }\end{array}$ & Prepare & 44 & $\begin{array}{l}\text { Move medication cart to } \\
\text { patient }\end{array}$ & Admin \\
\hline 10 & Prepare medications & Prepare & 45 & $\begin{array}{l}\text { Get keys and unlock } \\
\text { medication drawer }\end{array}$ & Admin \\
\hline 11 & Verify medication dosage & Prepare & 46 & $\begin{array}{l}\text { Walk from medication drawer } \\
\text { to medication cart }\end{array}$ & Admin \\
\hline 12 & Bring medication to patient & Admin & 47 & $\begin{array}{l}\text { Move medication cart out of } \\
\text { way }\end{array}$ & Admin \\
\hline 13 & $\begin{array}{l}\text { Scan patient identification } \\
\text { band }\end{array}$ & Admin & 48 & $\begin{array}{l}\text { Position COW (computer on } \\
\text { wheels) }\end{array}$ & Admin \\
\hline 14 & Chat with patient again & Admin & 49 & Dispose of trash & Admin \\
\hline 15 & Identify patient & Admin & 50 & Get supplies & Admin \\
\hline 16 & $\begin{array}{l}\text { Explain medication purpose } \\
\text { and action }\end{array}$ & Admin & 51 & Walk from floor to cart & Admin \\
\hline 17 & $\begin{array}{l}\text { Answer patient question } \\
\text { about medications }\end{array}$ & Admin & 52 & Unlock cart & Admin \\
\hline 18 & $\begin{array}{l}\text { Perform pre-administration } \\
\text { assessments }\end{array}$ & Admin & 53 & Relock cart & Admin \\
\hline 19 & Administer medications & Admin & 54 & Enter room & Admin \\
\hline 20 & Provide follow-up care & Admin & 55 & Get med cup & Admin \\
\hline 21 & $\begin{array}{l}\text { Document medication } \\
\text { administration }\end{array}$ & Document & 56 & $\begin{array}{l}\text { Cannot see but hear pill } \\
\text { dropped }\end{array}$ & Admin \\
\hline 22 & $\begin{array}{l}\text { Document patient } \\
\text { medications refusal }\end{array}$ & Document & 57 & Typing on key board & Admin \\
\hline 23 & Log-off MAR & Document & 58 & Check for allergies & Admin \\
\hline 24 & Bid farewell to patient & Document & 59 & Prepare meds for scan & Admin \\
\hline 25 & Post-wash hands & Document & 60 & Obtain medication again & Admin \\
\hline 26 & Answer phone & Interruption & 61 & Other $1^{*}$ & N/A \\
\hline 27 & Make a phone call & Interruption & 62 & Other $2^{*}$ & N/A \\
\hline 28 & Medication omission & Interruption & 63 & \multicolumn{2}{|c|}{ Document assessment findings Document } \\
\hline 29 & Notify physician & Interruption & 64 & Assess patient & Prepare \\
\hline 30 & Patient inquiry & Interruption & 65 & Look at ID band & Prepare \\
\hline 31 & Procedural error & Interruption & 66 & Other $3^{*}$ & N/A \\
\hline 32 & Staff inquiry & Interruption & 67 & Personal time & Interruption \\
\hline 33 & Knock on door & Prepare & 68 & At medication cart & Prepare \\
\hline 34 & Scan meds & Admin & 69 & Introduce self & Prepare \\
\hline 35 & MAR with unknown reason & Document & 70 & At computer & Prepare \\
\hline
\end{tabular}

*Unidentifiable activities due to view being obstructed. 


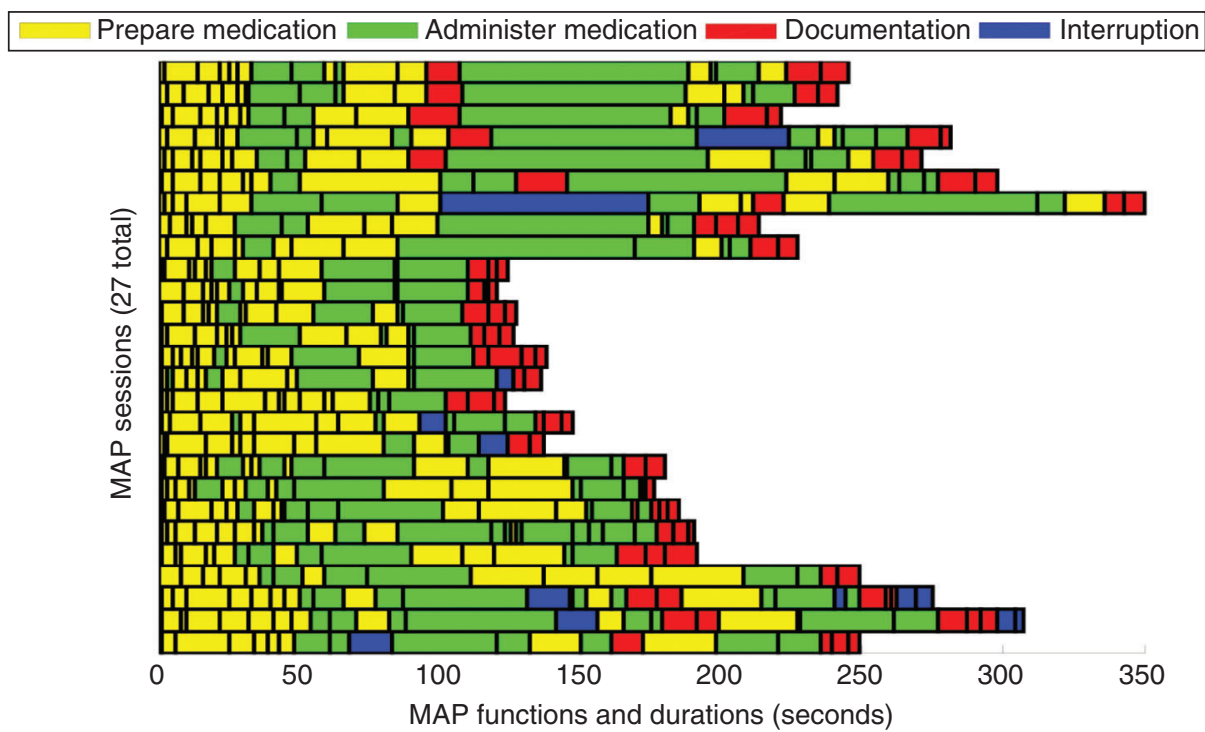

Figure 1. MAP workflow timeline belt.

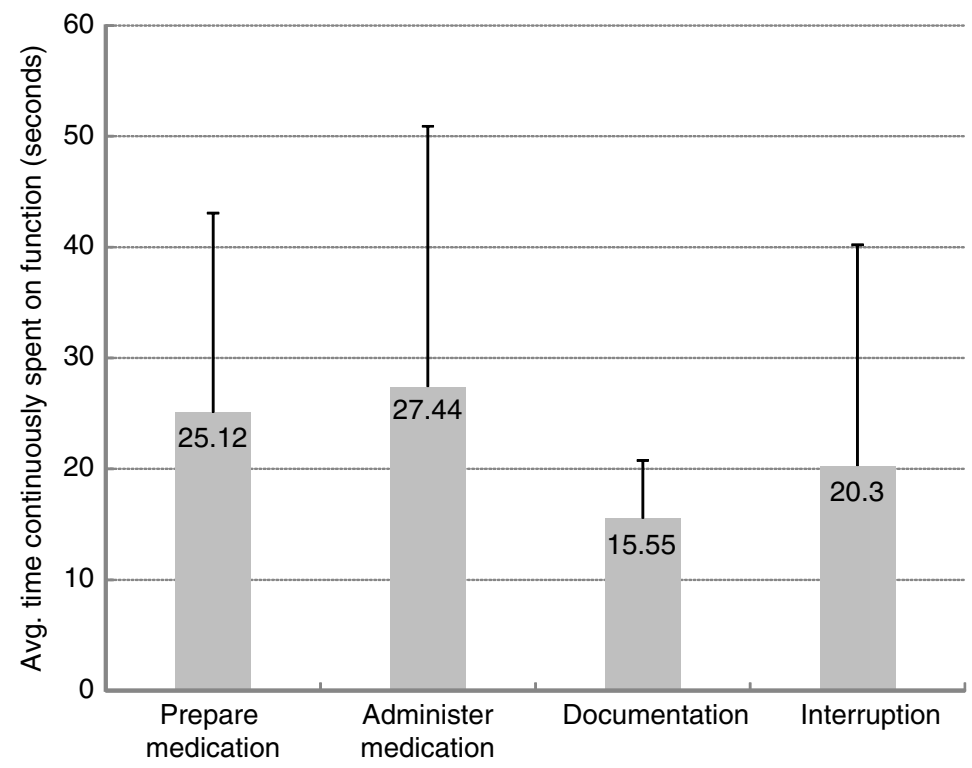

Figure 2. Average continuous function performance time.

medication administration had the longest average continuous time at 27.44 seconds and the largest standard deviation, while documentation had the shortest average continuous time at 15.55 seconds and the smallest standard deviation. The smaller standard deviation implied that there was less variability in completing that task. 
(a)

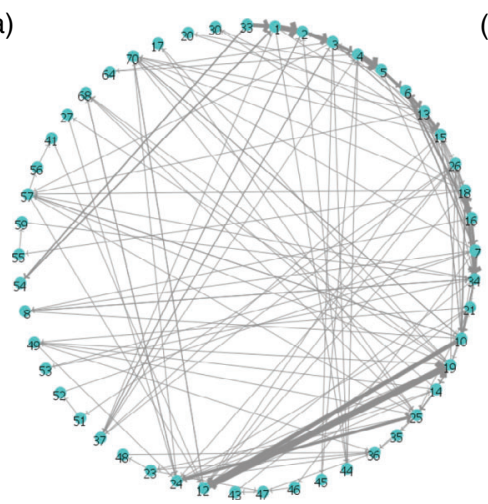

(b)

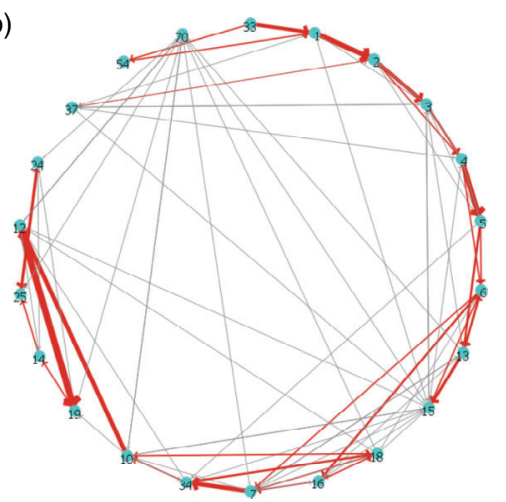

Figure 3. Network plots: (a) with all tasks; (b) with only tasks $\geq 15$ transitions.

MAP transition frequencies between pairs of tasks were examined using network plots [6]. Figures 3a and 3b show network plots of baseline MAP workflow with each node representing a task from Table 1 . The links represent transitions between pairs of tasks. The width of a link is proportional to the transition frequency between pairs of tasks. Figure $3 \mathrm{a}$ shows the transitions between all task pairs while Figure $3 \mathrm{~b}$ shows only task pairs with a minimum of 15 transitions to display the more frequent tasks and transitions. The more significant transitions are highlighted in red.

The network plot in Figure $3 b$ depicts several interesting patterns. For example, Tasks 33 (knock on door), 1 (greet patient), 2 (pre-wash hands) and 3 (chat with patient) generally occurred in sequence. Task 4 (scan patient ID band) often led to Task 5 (log-on to MAR). For Task 6 (review of MAR), a number of subsequent tasks occurred including Tasks 7 (obtain medications, 16 (explain medication purpose and action), and 15 (identify patient). Task 34 (scan medication) often followed Task 7 (obtain medication). Tasks 10 (prepare medication), 12 (bring medication to patient) and 19 (administer medication) generally occurred together. Tasks 24 (bid farewell to patient) and 25 (post-wash hands) were nearly interchangeable.

Figures 1, 3a and $3 \mathrm{~b}$ illustrate the nonlinear nature of MAP workflow. These data indicated that different RNs had different workflow patterns. Furthermore, an RN did not perform MAP functions and tasks in the same sequence from one patient to the next. The workflow inconsistencies suggested that RNs were making decisions in real-time and on a case-by-case basis in response to patient's needs and other demands. Therefore, an agent-based approach is most appropriate for development of an MAP computer simulation model that accurately portrays RN characteristics and behavior.

\subsection{MAP Simulation Model Development}

This section describes the process used to develop the MAP simulation model. The process steps include modeling approach, model development, model validation, and model testing. 


\subsubsection{Modeling Approach}

An agent-based modeling approach was taken to develop the MAP computer simulation model. The initial step was identification of the behavior of the RN agent based on natural MAP workflow sequences derived from MAP pilot study data (section 2). These workflow sequences were determined by calculating the task transition probabilities associated with the RN's movement from one task to another (Figure 3). This approach highlighted variations in MAP workflow patterns from one RN to the next and for a specific RN from patient to patient. Pairs of tasks with higher transition probabilities that were more likely to be performed together were also identified.

\subsubsection{Model Development}

The MAP model was developed from MAP baseline data using Netlogo [18], an agentbased computer simulation platform and programming language. The final model reflects the sequence of MAP functions and tasks performed by RNs while engaged in oral medication administration. Each RN was modeled as an independent agent administering oral medications to one patient at a time. Medication administration functions and tasks were based on specified probabilities derived from task transitions represented in baseline MAP data. Due to the small sample size, an average value was used instead of a best-fit distribution for task transitions. In the MAP model, when an RN began a task, he/she remained engaged in that task for the entire duration of the task. Upon completion of the task, the RN then moved on to the next task based on the transition probabilities. The amount of time that the RN spent on each task was based on baseline MAP data.

MAP model programming involved two key functionalities that corresponded to the Setup and Go+ buttons on the graphical user interface (GUI). As described by the program pseudo code displayed in Figure 4, the Setup function initializes the model and creates a graph using nodes and links as shown in Figure 5. The Go+ function has a

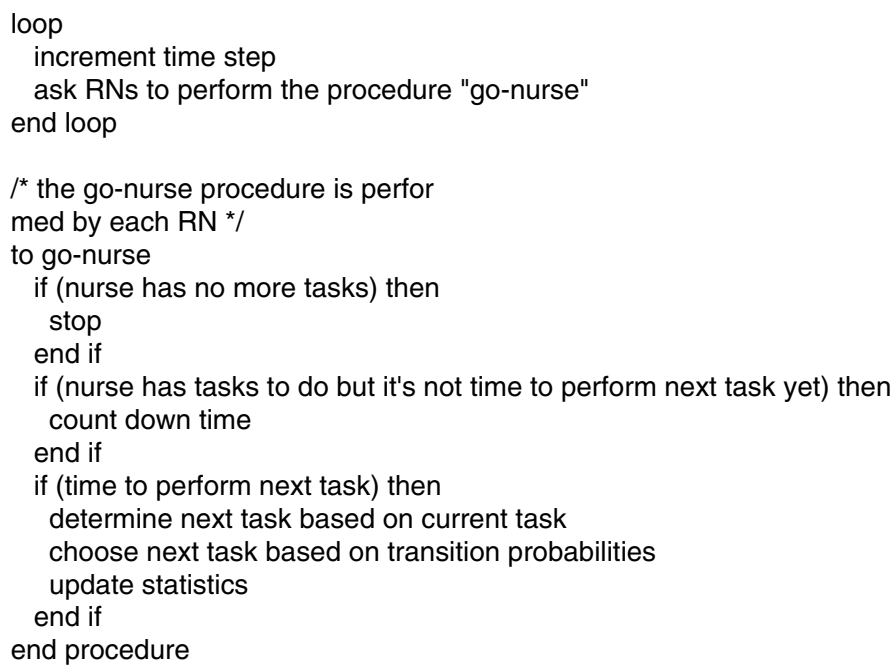

Figure 4. Agent-based MAP simulation model pseudo code. 


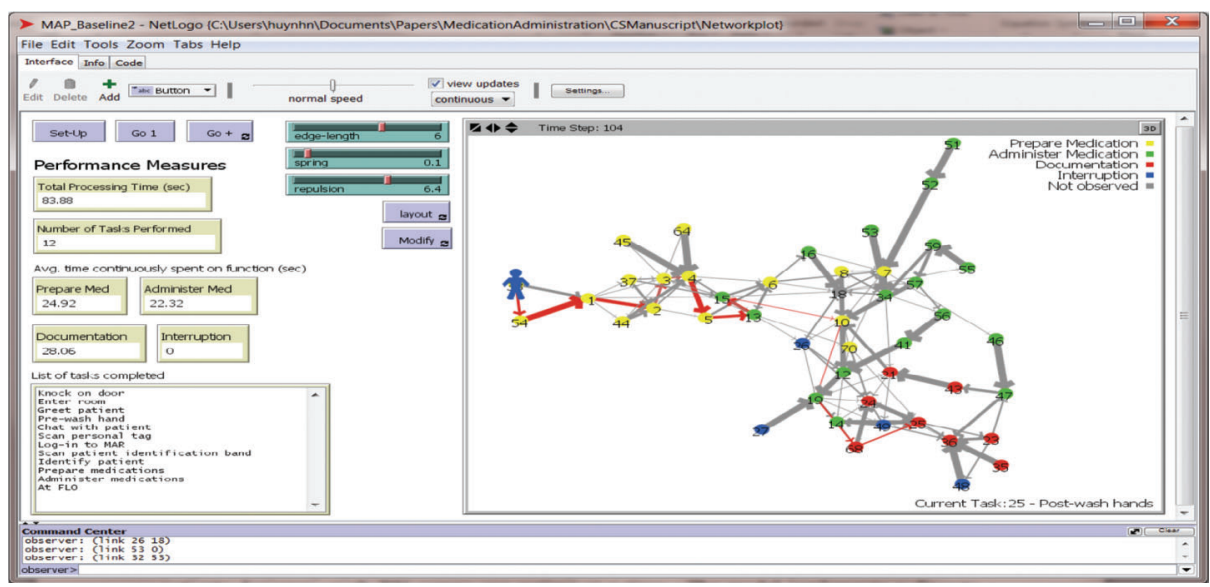

Figure 5. MAP simulation model graphical user interface.

main loop that is called at every time step or "tick." The "go-nurse" procedure contains the key logic of the MAP model. Specifically, each RN progresses to the next task based on transition probabilities. Once a task is selected, the RN is "busy" for the duration of the task. When the task is completed, the RN moves to the next task. This process is repeated until the $\mathrm{RN}$ has completed all tasks.

A graphical visualization technique was employed to depict the RN workflow pattern. As illustrated in the simulation model screenshot in Figure 5, the MAP model's GUI includes: (1) control buttons that allow users to run and reset the model, (2) an animation screen that illustrates the RN's workflow pattern, (3) graph configuration parameters, (4) an option to modify the graph for redesign evaluation purposes, and (5) performance measures including total patient processing time and time continuously spent on different functions.

\subsubsection{Model Validation}

MAP model validation was achieved by comparing the model's outcome variables with baseline MAP observation data. The two outcome variables used to validate the model were (1) the amount of time spent performing a function, and (2) the variation in the number of tasks performed. An F-test was conducted to examine the equivalence of the variance between the model data and baseline data. A t-test was then performed to test two null hypotheses: (1) the difference in the means of the total patient processing time between the model and baseline MAP observation data is zero $\left(\mathrm{H} 1_{0}\right)$, and (2) the difference in the means of the number of tasks performed between the model and baseline MAP observation data is zero $\left(\mathrm{H} 2_{0}\right)$.

\subsubsection{Model Testing}

The validated MAP simulation model was used to test two what-if scenarios: (1) would the average time spent continuously on performing the medication preparation function improve if an RN were to perform selected MAP tasks in sequence? and (2) would the 
variation in the number of tasks performed and the amount of time spent performing the functions change if an RN were to perform tasks in the order most frequently observed, i.e., highest two transition probabilities? To test scenario 1 , the validated model was altered to create an MAP Redesign Model 1 that sequenced tasks 1-6 together, i.e., greet patient (task 1), pre-wash hands (task 2), chat with patient (task 3), scan patient ID band (task 4), log-in to medication administration record (MAR) (task 5), and review MAR (task 6), rather than the observed random sequence captured in MAP baseline data and depicted in Figure 3b. To test scenario 2, the validated model was altered to create an MAP Redesign Model 2 in which tasks were selected based on the two highest transition probabilities rather than the observed workflow variations displayed in MAP baseline data. In this scenario, only the two most dominant tasks (those with two highest transition probabilities) were retained. It should be noted that the resulting workflow sequence could be different from one simulation run to another.

\section{RESULTS}

The MAP model validation results are summarized in Table 2. The t-test was employed to assess the presented hypotheses $\left(\mathrm{H}_{0}\right.$ and $\mathrm{H} 2_{0}$ defined in Table 2). Specifically, the ttest with equal variance is performed after F-test results supported acceptance of the equal variance assumption. As shown in Table 2 , the t-test of $\mathrm{H} 1_{0}$ using the statistical software $\mathrm{R}$ [19] yielded $\mathrm{t}=0.94, \mathrm{df}=1025, \mathrm{p}$-value $=0.3487$. Given that the $\mathrm{p}$-value was greater than the significance level (0.05), the null hypothesis could not be rejected. In other words, the model gave a total patient processing time that is comparable with the observed data. Similarly, a t-test was performed to test $\mathrm{H} 2_{0}$. Results of the t-test were $\mathrm{t}=-1.13$, df $=1025$, $\mathrm{p}$-value $=0.2582$. Thus, the model provided a comparable number of tasks performed to observation data.

As reflected in Table 3, for MAP Redesign Model 1, the average time spent continuously on medication preparation increased from 22 to 30 seconds. To determine whether this increase was statistically significant, a t-test was performed with the null hypothesis being that the difference in means between the baseline model and the Redesign Model 1 model was zero. Results of the t-test yielded $t=-16.68, \mathrm{df}=1998$, $\mathrm{p}$-value $<0.0001$. Given that the p-value was less than the significance level (0.05), the null hypothesis was rejected. In other words, the true difference in means was not equal to 0 and thus there was a significant benefit to sequencing Tasks $1-6$. It was interesting to note that, although the average time continuously spent on the different functions was higher for the Redesign

Table 2. Model validation results

\begin{tabular}{lcccc}
\hline \multicolumn{1}{c}{ Hypothesis } & t-value & Degree of freedom & p-value & Decision \\
\hline $\begin{array}{l}\mathrm{H} 1_{0} \text { : simulation model mean patient } \\
\text { processing time }=\text { observed mean } \\
\text { patient processing time }\end{array}$ & 0.94 & 1025 & 0.3487 & Fail to \\
H2 $2_{0}$ : simulation $\mathrm{H} 1_{0}$ \\
$\begin{array}{l}\text { tasks performed by } \mathrm{RN}=\text { observed } \\
\text { mean number of tasks performed by } \mathrm{RN}\end{array}$ & -1.13 & & & Fail to \\
\end{tabular}


Table 3. Impact of MAP redesign

\begin{tabular}{lccc}
\hline Performance Measure & $\begin{array}{c}\text { MAP } \\
\text { baseline }\end{array}$ & $\begin{array}{c}\text { MAP } \\
\text { redesign 1 }\end{array}$ & $\begin{array}{c}\text { MAP } \\
\text { redesign 2 }\end{array}$ \\
\hline Avg. total processing time (sec) & 191 & 193 & 195 \\
Avg. number of tasks performed & 21 & 21 & 20 \\
Avg. time continuously spent on preparing medication & & & \\
(sec) & 22 & 30 & 20 \\
Avg. time continuously spent on administering & 18 & 22 & 18 \\
medication (sec) & 30 & 32 & 39 \\
Avg. time continuously spent on documentation (sec) & 17 & 18 & 0 \\
Avg. time continuously spent on interruption (sec) & & &
\end{tabular}

Model 1 compared to the baseline model, the total processing time for the Redesign Model 1 was not significantly different from the baseline model ( $\mathrm{p}$-value $=0.5252$ ). This result suggested that some workflow standardization which allows nurses to stay focused on the function at hand does not impact the total patient processing time.

In MAP Redesign Model 2, the average number of tasks performed decreased from 21 to 20 (Table 3). As shown in Figure 6, the variance in the number of tasks performed in MAP Redesign Model 2 was smaller than that of the baseline model. In addition, the variation of the number of tasks in MAP Redesign Model 2 was smaller than that in the baseline model. From Table 3, it can be seen that the average time continuously spent on medication preparation ( $22 \mathrm{vs} .20 \mathrm{sec}$ ) and administration (18 vs. $18 \mathrm{sec}$ ) did not increase as a result of MAP redesign. However, the average time continuously spent on documentation increased significantly from 30 to $39 \mathrm{sec}$. The t-test confirmed that the true difference in means was not equal to 0 time $(\mathrm{t}=-19.58 \mathrm{df}=1854.941$, $\mathrm{p}$-value $<0.0001)$ suggesting that MAP redesign had a key impact on medication administration documentation.

\section{DISCUSSION}

The MAP computer simulation model provided a foundational framework for assessing MAP redesign options. Initial testing of the basic redesign capabilities of the model indicated that it has the potential to support modeling of more complex MAP functions and tasks. A unique feature of the computer simulation is the ability to engage clinicians in low-risk pre-implementation MAP redesign. As illustrated in Figure 5, the model shows clinicians their current MAP workflow sequence through a red highlighted path on the display graph. This graph technique allows clinicians to easily identify their current MAP workflow and assess problem areas that are having an impact on MAP efficiency, quality, and safety. Clinicians can then add or delete model graph nodes, i.e., tasks, to simulate MAP redesign options to assess their potential impact on the MAP.

While results highlight preliminary feasibility for use of computer simulation in MAP redesign, important limitations underscore the need to continue model refinement and testing. First, the modeling was based on a very small sample of observations. Second, the simplistic oral medication administration process did not capture the 


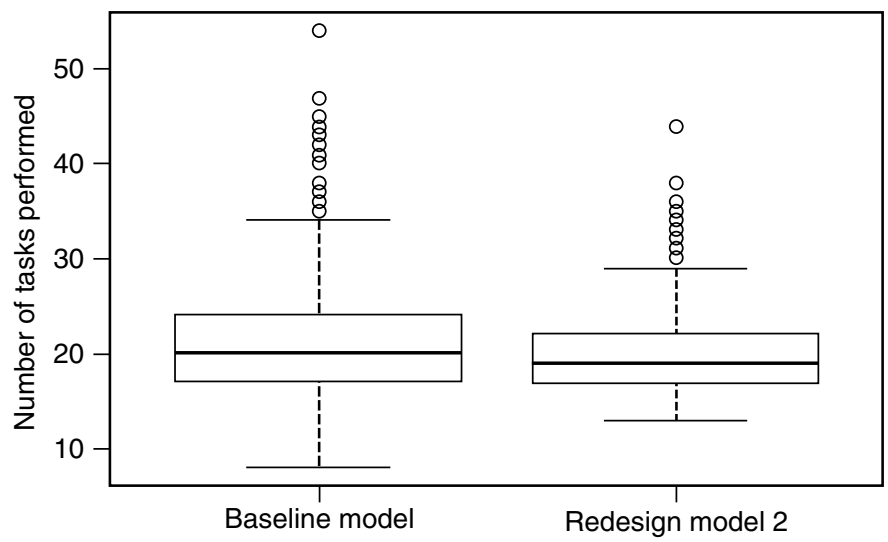

Figure 6. Task comparison between MAP baseline model and MAP redesign model 2.

Table 4. Selected examples of anticipated clinical MAP interruptions

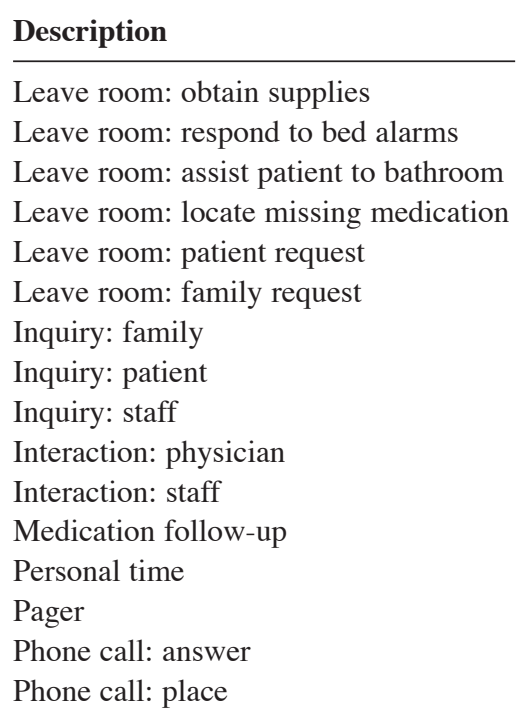

complexity that surrounds actual medication administration processes. Finally, the oral medication administration process was modeled in a simulated environment that was not characterized by the typical interruptions that occur in a complex patient care unit. Additional research is currently underway to collect MAP data in an actual clinical setting using observation tools and methods established in previous pilot studies. It is anticipated that these clinical MAP data will provide a more accurate assessment of workflow fragmentation resulting from interruptions. Selected examples of anticipated clinical interruptions are provided in Table 4 . Such behavioral data will be incorporated into the agent-based computer simulation model to enhance its validity. 


\section{CONCLUSION}

This paper describes the development and testing of a computer simulation model as a tool to support low-risk redesign of the MAP. Preliminary findings indicated that the tool provides a reliable, valid and feasible approach to redesign of the complex MAP. In particular, the use of an agent-based modeling approach and actual MAP workflow data enhanced the potential of the tool to support MAP redesign. However, a number of important limitations underscore the need for further testing of the tool with complex clinical data, especially those factors that contribute to workflow fragmentation such as interruptions. This will provide more complex behavioral and social contextual factors that better represent real-world MAP dynamics.

\section{ACKNOWLEDGMENTS}

This work benefitted significantly from the assistance of the RNs who provided their expertise as MAP models and the nurse subject matter experts who engaged in the time consuming work of MAP video recording evaluation. Their dedication to the project is greatly appreciated. The authors are also appreciative of Ms. Gene Meding for her project support.

\section{CONFLICT OF INTEREST}

The author indicated no potential conflicts of interest.

\section{REFERENCES}

[1] Aspden P, Wolcott J, Bootman JL, Cronenwett LR. Preventing, Medication Errors: Quality Chasm Series. Institute of Medicine, Washington D.C., 2007.

[2] Burke K. Executive summary: the state of the science on safe medication administration symposium. Journal of Infusion Nursing. 2005, 28(Suppl 2):4-9.

[3] Hughes R, Ortiz E. Medication errors: Why they happen, and how they can be prevented. American Journal of Nursing. 2005, 105(3):14-24.

[4] Sullivan C, Gleason KM, Rooney D, Groszek JM, Barnard, C. Medication reconciliation in the acute care setting: opportunity and challenge for nursing. Journal of Nursing Care Quality. 2005, 20(2):95-98.

[5] Carayon P, Hundt AS, Karsh BT, Gurses AP, Alvarado CJ, Smith M, Brennan PF. Work system design for patient safety: The SEIPS model. Quality and Safety in Health Care. 2006, 15(Suppl 1):i50-i58.

[6] Zheng K, Haftel, HM, Hirschl RB, O'Reilly M, Hanauer DA. Quantifying the Impact of Health IT Implementations on Clinical Workflow: a New Methodological Perspective. Journal of the American Medical Informatics Association. 2010, 17:454-461.

[7] Grigg SJ, Garrett SK, Craig JB. A Process Centered Analysis of Medication Administration: Identifying Current Methods and Potential for Improvement. International Journal of Industrial Ergonomics. 2011, 41(4):380-388.

[8] McBride-Henry K, Foureur M. Medication administration errors: Understanding the issues. Australian Journal of Advanced Nursing. 2006, 23(3):33-42.

[9] Tang, FI, Sheu SJ, Yu S, Wei, IL, Chen CH. Nurses relate the contributing factors involved in medication errors. Journal of Clinical Nursing. 2007, 16(3):447-457.

[10] Karr T. Determining what healthcare should be. Industrial Engineer. 2011, 23(9):45-48.

[11] Reid PP. Building a better delivery system: A new engineering/health care partnership. The National Academies Press: Washington D.C. 2005. 
[12] Institute of Medicine. To err is human: Building a safer health system. National Academy Press, Washington D.C., 1999.

[13] Institute of Medicine. Crossing the quality chasm: A new health system for the $21^{\text {st }}$ century. National Academy Press, Washington D.C., 2001.

[14] Institute of Medicine. Keeping patients safe: Transforming the work environment of nurses. National Academies Press, Washington D.C., 2004.

[15] Sibbel R, Urban C. Agent-Based Modeling and Simulation for Hospital Management. Cooperative Agents: Applications in the Social Sciences (eds. Saam and Schmidt). Kluwer Academic Publishers, Netherlands, 2001

[16] Kanagarajah, AK, Lindsay P, Miller A, Parker D. An Exploration into the Uses of Agent-Based Modeling to Improve Quality of Health Care. Proceedings of the $6^{\text {th }}$ International Conference on Complex Systems. 2006, 1-10.

[17] Snyder R, Huynh N, Cai B, Tavakoli AS. Medication Administration Process Evaluation: Tools and Techniques. Journal of Healthcare Engineering. 2011, 2(4):527-538.

[18] Wilensky U. Netlogo itself. Center for Connected Learning and Computer-Based Modeling, Northwestern University, Evanston, IL. 1999. http://ccl.northwestern.edu/netlogo/. Accessed August 22, 2011.

[19] R Development Core Team. R: A language and environment for statistical computing. R Foundation for Statistical Computing, Vienna, Austria. 2007. http://www.R-project.org. Accessed August 26, 2011. 


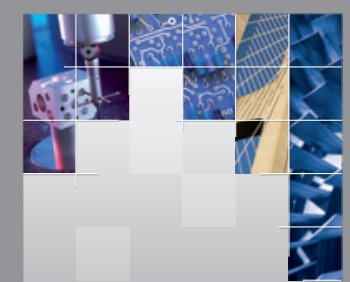

\section{Enfincering}
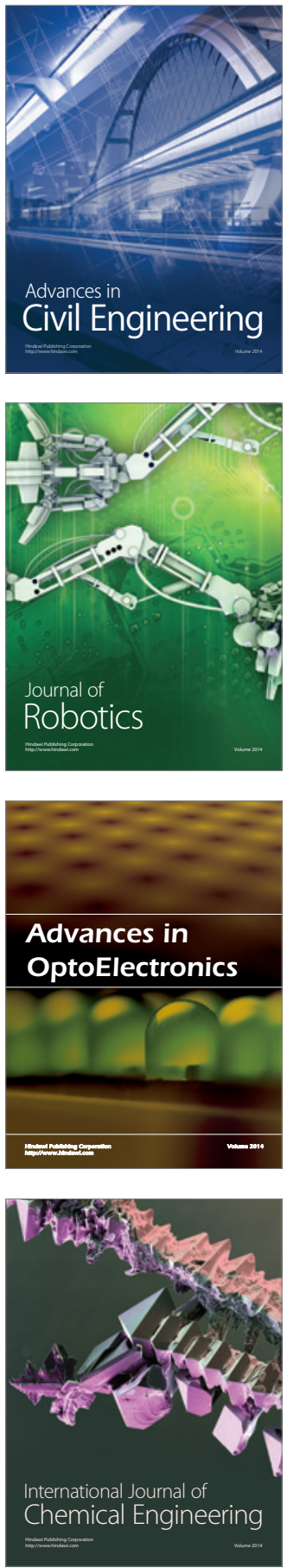

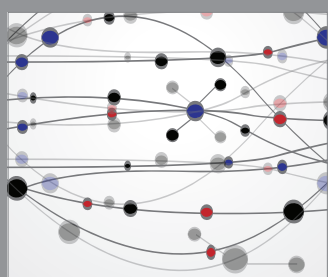

The Scientific World Journal

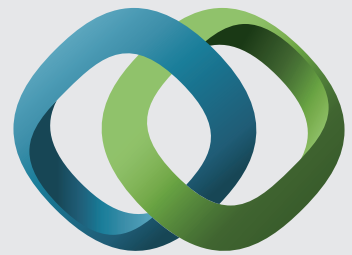

\section{Hindawi}

Submit your manuscripts at

http://www.hindawi.com
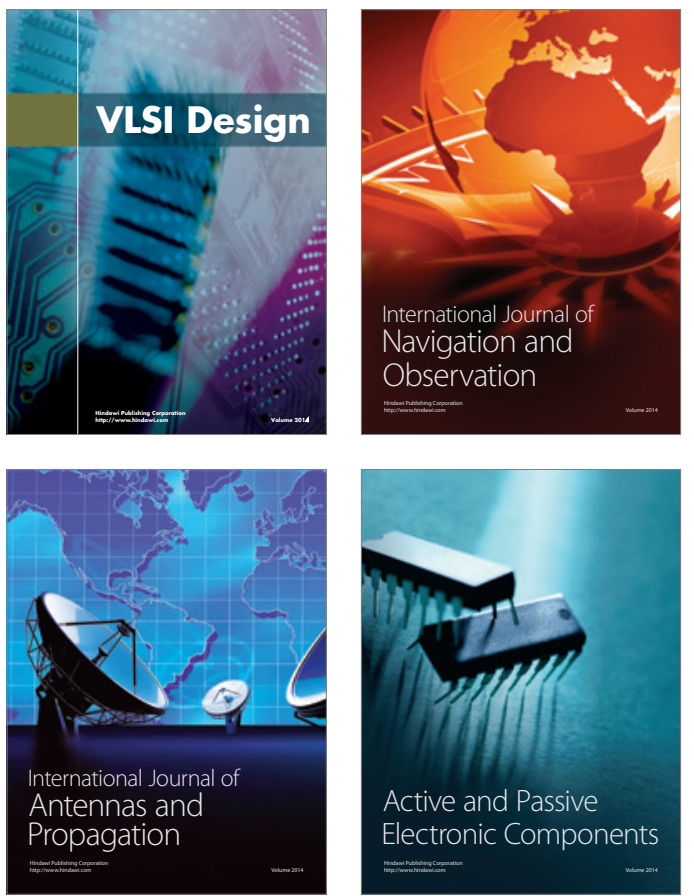
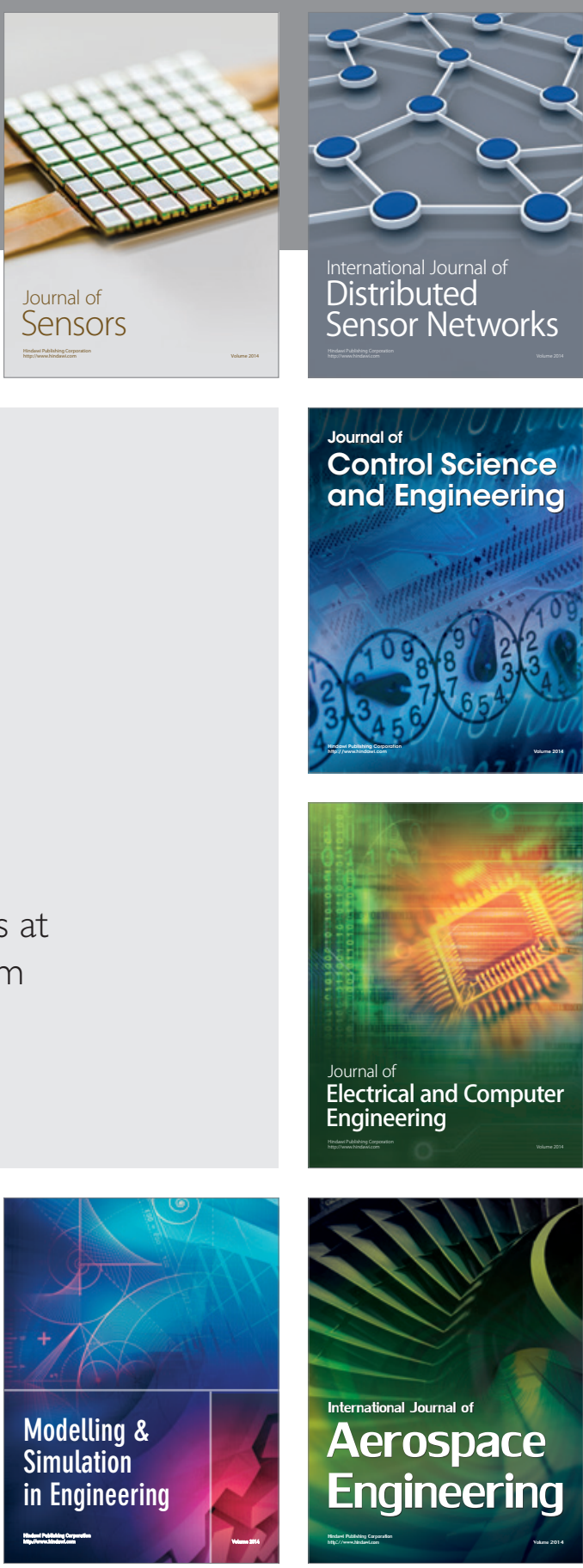

International Journal of

Distributed

Sensor Networks

Journal of

Control Science

and Engineering
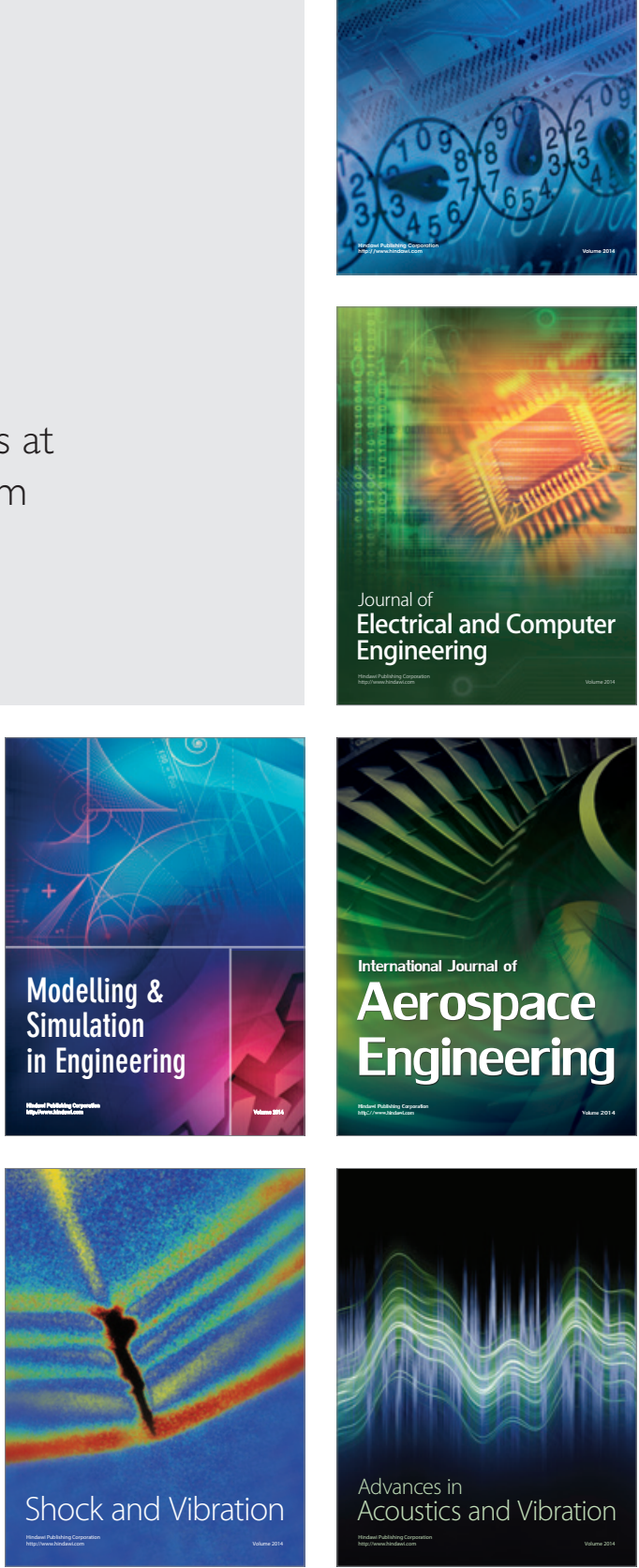